INPLASY

PROTOCOL

To cite: Pang et al.

Effectiveness and safety of aromatherapy massage for knee Osteoarthritis: A protocol for systematic review and meta analysis. Inplasy protocol

202210010. doi:

10.37766/inplasy2022.1.0010

Received: 02 January 2022

Published: 02 January 2022

Corresponding author:

Yufeng Wang

wangchn@126.com

Author Affiliation:

Changchun University of

Chinese Medicine.

Support: None.

Review Stage at time of this submission: Preliminary searches.

Conflicts of interest:

None declared.

\section{Effectiveness and safety of aromatherapy massage for knee Osteoarthritis: A protocol for systematic review and meta analysis}

Pang, T1; Liu, C2; Li, J3; Yao, J4; Wang, Y5.

Review question / Objective: Population: adults with osteoarthritis of the knee; Intervention: aromatherapy massage (aromatherapy massage with essential oils as a medium); Comparison: other treatment; Outcome: the Western Ontario and McMaster Universities Osteoarthritis Index scale.

Information sources: Related studies in the following databases will be searched from inception to December 30 , 2021: PubMed, Embase, Web of Science, the Cochrane Library, China National Knowledge Infrastructure, VIP and Wanfang.

INPLASY registration number: This protocol was registered with the International Platform of Registered Systematic Review and Meta-Analysis Protocols (INPLASY) on 02 January 2022 and was last updated on 02 January 2022 (registration number INPLASY202210010).

\section{INTRODUCTION}

Review question / Objective: Population: adults with osteoarthritis of the knee; Intervention: aromatherapy massage (aromatherapy massage with essential oils as a medium); Comparison: other treatment; Outcome: the Western Ontario and McMaster Universities Osteoarthritis Index scale.

Condition being studied: Knee osteoarthritis (KOA) is a chronic degenerative disease with pain as the main symptom that seriously affects the quality of life of middle-aged and elderly people. Studies have shown that the risk of lower 
limb disability due to KOA is at least $40 \%$ in the elderly, and with the increasing aging of the global population, KOA has become a serious public health problem that needs to be addressed in society. Currently, there is no effective radical treatment for KOA, and the main goal is to relieve joint pain and improve joint function symptoms. Aromatherapy massage is one of the widely popular complementary and alternative medical interventions, i.e., the use of various techniques on the human body surface and meridians or acupuncture points, using aromatic substances as the medium for transdermal delivery of massage therapy, in order to achieve the purpose of strengthening the body and treating diseases. Some studies have shown that aromatherapy massage can reduce pain, fatigue, anxiety and sleep disorders, and now clinical studies have proved that aromatherapy massage can reduce pain, improve functional status and enhance the quality of life for KOA patients. After our preliminary study, we found that there is no systematic review on whether aromatherapy massage is safe and effective for patients diagnosed with KOA; therefore, we used this protocol to comprehensively evaluate the efficacy of aromatherapy massage on KOA.

\section{METHODS}

Participant or population: All patients with KOA will be included without limitation of age, race, sex, economic level, and severity.

Intervention: The interventions in the experimental group included only aromatherapy massage. It mainly included different single oil massage and compound oil massage, etc. There was no restriction on the method, time and frequency of massage in the experimental group.

Comparator: Controlled interventions included control groups with no treatment, sham/placebo groups, or other conventional treatments.
Study designs to be included: Only randomized controlled trials (RCTs) will be included in this study.

Eligibility criteria: We will include only the literature of randomized controlled trials (RCTs) of aromatherapy massage for KOA. Nonrandomized controlled studies case reports, case series and reviews will not be included in this study.

Information sources: Related studies in the following databases will be searched from inception to December 30, 2021: PubMed, Embase, Web of Science, the Cochrane Library, China National Knowledge Infrastructure, VIP and Wanfang.

Main outcome(s): The primary outcome was scales of The Western Ontario and McMaster Universities Osteoarthritis Index scale.

Additional outcome(s): Secondary endpoints included Visual analog scale, Symptom score, Lysholm knee scoring scale, Adverse events.

Quality assessment / Risk of bias analysis: The quality of all RCTs will be evaluated with the Cochrane Collaboration tool. Two authors (Junjie Yao and Chang Liu) will independently conduct quality evaluations, and any controversy will be addressed by discussion with another author (Tingting Pang).

Strategy of data synthesis: The metaanalysis of data from included outcomes will be performed using the RevMan V.5.4.1, and we will choose a randomized or fixed effect model for data statistics according to the results of the heterogeneity test. The enumeration data were expressed as relative risk (RR), and the weight mean difference (WMD) was used as the measurement data; each effect amount was expressed in $95 \%$ confidence interval (Cl). The specific methods were as follows: If the heterogeneity was low $\left(I^{2}<50 \%\right.$, the fixed-effects model was used for data synthesis. If there is high heterogeneity $\left(I^{2}>\right.$ $50 \%$ ), the random-effects model will be used for data synthesis after excluding 
possible heterogeneity sources. The investigation methods included subgroup and sensitivity analyses. If data cannot be synthesized, we provide a descriptive analysis to solve this problem.

Subgroup analysis: If there was high heterogeneity $(12>50 \%)$ among the included studies, we conducted a subgroup analysis to analyze the sources of heterogeneity according to the following factors: age, sex, race, courses, sample sizes, different methods of aromatherapy massage, and other possible factors affecting the results. If necessary, we will conduct a subgroup analysis of the different doses of the drug, treatment time, different subtypes of KOA.

Sensitivity analysis: To test the stability and reliability of the results of this study, we conducted a sensitivity analysis according to the following points: method quality, sample size, and missing data. After that, we will perform a data analysis again and compare the results. If there was no directional change after the sensitivity analysis, the results were stable.

Language: English.

Country(ies) involved: China.

Keywords: Aromatherapy massage; Knee Osteoarthritis; Systematic review; Metaanalysis.

Contributions of each author:

Author 1 - Tingting Pang.

Email: 815483770@qq.com

Author 2 - Chang Liu.

Author 3 - Jiahui Li.

Author 4 - Junjie Yao.

Author 5 - Yufeng Wang.

Email: wangchn@126.com 